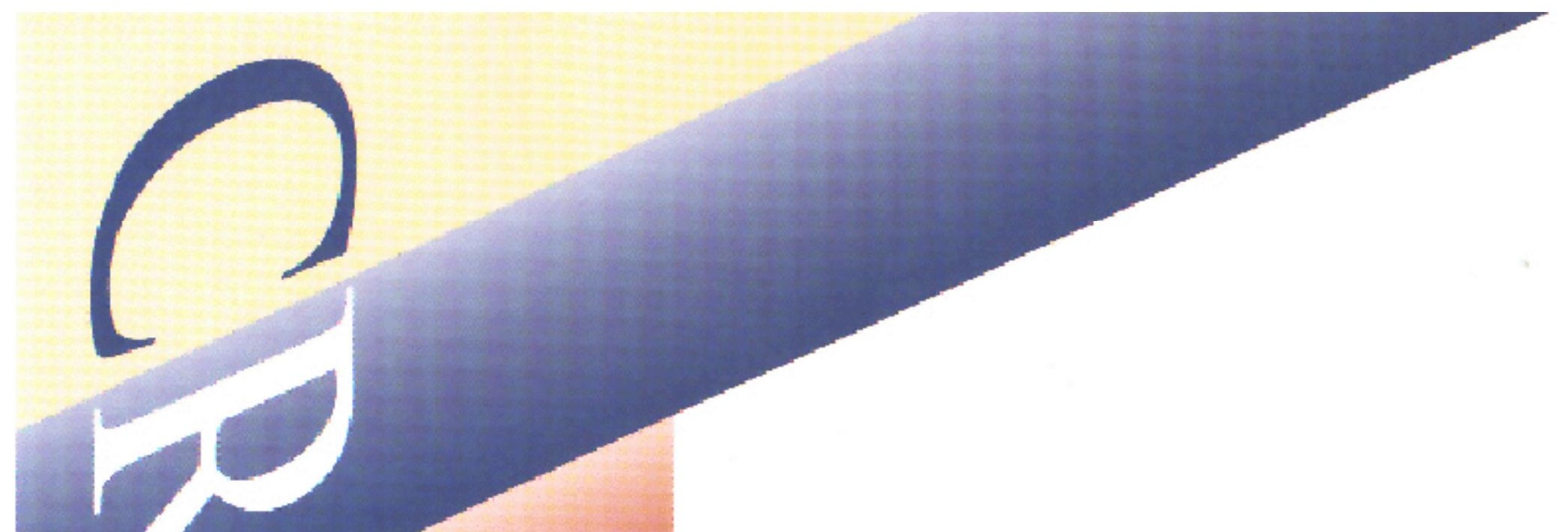

CAHIER 10-2002

EFFICIENT AND NON-DETERIORATING CHOICE

Walter BOSSERT and Yves SPRUMONT

Centre de recherche et développement en économique

C.P. 6128, succursale Centre-ville Montréal QC H3C 3J7

Téléphone : (514) 343-6557

Télécopieur : (514) 343-5831

crde@crde.umontreal.ca

http://www. crde. umontreal.ca/

Université $\mathbf{t h}$ de Montréal 
CAHIER 10-2002

\title{
EFFICIENT AND NON-DETERIORATING CHOICE
}

\author{
Walter BOSSERT ${ }^{1}$ and Yves SPRUMONT ${ }^{1}$
}

1 Centre de recherche et développement en économique (C.R.D.E.) / Centre interuniversitaire de recherche en économie quantitative (CIREQ) and Département de sciences économiques, Université de Montréal

May 2002

The authors thank Kotaro Suzumura for discussions and comments. Financial support from the Social Sciences and Humanities Research Council of Canada and the Fonds pour la formation de chercheurs et l'aide à la recherche of Québec is gratefully acknowledged. 


\section{RÉSUMÉ}

A chaque problème de choix constitué d'un ensemble d'options réalisables et d'un statu quo, une règle de sélection fait correspondre un sous-ensemble des options réalisables. Nous étudions la "rationalisabilité" collective de telles règles. Plus précisément, nous analysons les conditions sous lesquelles il existe $n$ relations d'ordre par rapport auxquelles les options sélectionnées sont efficaces et dominent le statu quo.

Mots clés : choix social, efficacité, rationalité

\section{ABSTRACT}

We analyze collective choice procedures with respect to their rationalizability by means of profiles of individual preference orderings. A selection function is a generalization of a choice function where selected alternatives may depend on a reference (or status quo) alternative in addition to the set of feasible options. Given the number of agents $n$, a selection function satisfies efficient and non-deteriorating $n$-rationalizability if there exists a profile of $n$ orderings on the universal set of alternatives such that the selected alternatives are (i) efficient for that profile, and (ii) at least as good as the reference option according to each individual preference. We analyze efficient and nondeteriorating collective choice in a general abstract framework and provide a characterization result given a universal set domain.

Key words : social choice, efficiency, individual reationality 


\section{Introduction}

This paper contributes to a recent line of research that seeks to identify the testable restrictions of various theories of multi-agent decision-making. The approach is clearly in the spirit of the classical revealed preference analysis of choice initiated by Samuelson (1938, 1948), Arrow (1959), Richter (1966, 1971), Hansson (1968), Sen (1971) and others. However, while most earlier contributions in this area are concerned with the rationalizability of observed choices by means of a single (individual or social) preference relation, we ask whether there exists a profile of preference orderings-one ordering for each individual - such that the observed choices are obtained for this (not necessarily unique) profile according to the collective choice theory to be tested. In that respect, the approach of this paper resembles the analysis of Banerjee and Pattanaik (1996) who also deal with notions of rationality involving multiple preferences. The central question is: if preferences are not observable, how can we determine whether a given set of actual collective choices is consistent with a particular theory? More precisely, can we formulate necessary and sufficient conditions under which the observed behavior of the agents is rationalizable by some profile of preferences for the theory under examination?

While the question is relevant for all theories of collective choices, the existing literature has mostly focused on non-cooperative equilibrium concepts. In particular, Sprumont (2000) examines necessary and sufficient for Nash rationalizability, defined as follows. Suppose we can observe a collection of feasible sets of actions and a set of observed outcomes for each feasible set. These observations are Nash rationalizable if there exists a profile of preferences defined on combinations of these actions such that, for each game defined by a set of feasible actions and the restriction of these preferences to the associated combinations of feasible actions, the set of observed outcomes consists of the set of Nash equilibria of the game. Ray and Zhou (2001) perform a similar exercise for subgameperfect equilibria.

Here, we analyze two notions that play an elementary but fundamental role in essentially all cooperative approaches to collective choices: (Pareto) efficiency and individual rationality. Efficiency is well defined as soon as agents are assumed to possess preferences. Individual rationality, on the other hand, supposes the existence of a status quo or reference alternative: the requirement is that no agent should be worse off at a selected outome than at this status quo. From now on, we call such choices "non-deteriorating" to avoid any confusion with the widespread interpretation of rationality as preference-maximizing behavior. Understanding efficient and non-deteriorating behavior is a modest but essen- 
tial step towards a complete study of solution concepts such as the core (see Bossert and Sprumont, 2002) and (selections from) the set of Walrasian equilibria in an economy (see Brown and Matzkin, 1996).

We explore the testable restrictions of these hypotheses in a simple yet plausible framework. A selection function assigns, to each pair constiting of a feasible set of alternatives (included in some universal set) and a reference alternative (belonging to the feasible set), a subset of that feasible set. Alternatives have no particular structure and feasible sets are not restricted. This very general abstract model is developed by Zhou (1997) and also used in Bossert and Sprumont (2001) and Masatli and Ok (2002). While these papers are concerned with individual behavior, the model itself is perfectly suited to the analysis of collective choices as well. In an $n$-agent society, we call a selection function efficient and non-deteriorating (or, more precisely, $E N D_{n}$-rationalizable) if there exists a profile of preference orderings, one for each of the $n$ agents, according to which all selected alternatives are Pareto-efficient and at least as good as the status quo for every agent.

The implications of either of these two requirements are known. Bossert and Sprumont (2001) characterize non-deteriorating behavior, both in an abstract framework analogous to the one considered here and in a more structured economic environment. Efficiency by itself, on the other hand, imposes no restriction (if $n \geq 2$ ) because two agents may be assumed to have opposite preferences, in which case all alternatives in the universal set are efficient. The interplay between the two requirements, however, turns out to be interestingly complex: when added to the non-deterioration condition, efficiency generates substantial additional restrictions.

Much in the spirit of Richter (1966), we begin by identifying an END-congruence property that is necessary for $\mathrm{END}_{n}$-rationalizability. Our first theorem states that this condition is also sufficient if the cardinality of the universal set is smaller than $2(n+1)$. Moreover, for universal sets with $2(n+1)$ or more elements, there exist selection functions that are END-congruent but not $\mathrm{END}_{n}$-rationalizable. Our second theorem focuses on

the case where only the reference alternative varies: it asserts that the END-congruence property is also sufficient for any cardinality when all feasible sets under consideration coincide with the universal set.

\section{Arbitrary Domains}

The set of positive integers is denoted by $\mathbb{N}$. Let $X$ be a nonempty universal set, and let $N=\{1, \ldots, n\}$ with $n \in \mathbb{N}$ be the set of agents. $\mathcal{P}(X)$ is the power set of $X$ excluding the 
empty set. The cardinality of $X$ is denoted by $|X|$. A quasi-ordering on $X$ is a reflexive and transitive relation $R \subseteq X \times X$, and an ordering is a complete quasi-ordering. A binary relation $R$ on $X$ is antisymmetric if $(x R y$ and $y R x)$ implies $x=y$ for all $x, y \in X$. For notational convenience, we write $x R y$ instead of $(x, y) \in R$. Let $R_{d}$ be the diagonal on $X$, that is, for all $x, y \in X$,

$$
x R_{d} y \Leftrightarrow x=y
$$

The inverse $R^{-1}$ of a binary relation $R$ on $X$ is defined by

$$
x R^{-1} y \Leftrightarrow y R x
$$

for all $x, y \in X$. The transitive closure $\bar{R}$ of a binary relation $R$ on $X$ is defined as follows: for all $x, y \in X, x \bar{R} y$ if there exist $K \in \mathbb{N}$ and $x^{0}, \ldots, x^{K} \in X$ such that $x=x^{0}, x^{k-1} R x^{k}$ for all $k \in\{1, \ldots, K\}$ and $x^{K}=y$.

We consider collective choice problems where, in addition to a feasible set of alternatives, a reference alternative is part of the description of a choice situation. This reference alternative, which is assumed to be an element of the feasible set, can be interpreted as the status quo or the currently realized alternative in an intertemporal sequence of choices. In more structured environments such as exchange economies where the alternatives are allocations, the reference alternative could be a distribution of the total endowments in the economy.

Formally, a selection problem is a pair $(S, y)$ such that $S \in \mathcal{P}(X)$ and $y \in S$. The domain of selection problems is $\mathcal{D} \neq \emptyset$. A selection function is a mapping $C: \mathcal{D} \rightarrow \mathcal{P}(X)$ such that $C(S, y) \subseteq S$ for all $(S, y) \in \mathcal{D}$.

A selection function satisfies efficient and non-deteriorating rationalizability if there exists a profile of orderings on the universal set of alternatives such that the selected alternatives are (i) efficient for that profile, and (ii) at least as good as the reference option according to each individual preference. Because any selection function is rationalizable in that sense if we permit the degenerate case of the universal indifference relation, we require the components of the rationalizing profile to be antisymmetric; see also Bossert and Sprumont (2001).

Clearly, the existence of a profile rationalizing $C$ in the above sense depends on the number of individuals in a society. Therefore, we use the following definition of efficient and non-deteriorating (END) $n$-rationalizability of a selection function $C$.

END $_{n}$-Rationalizability: There exists a profile $\left(R_{1}, \ldots, R_{n}\right)$ of antisymmetric orderings 
such that, for all $(S, y) \in \mathcal{D}$ and for all $x \in C(S, y)$,

$$
x R_{i} y \text { for all } i \in N
$$

and

$$
\left\{z \in S \backslash\{x\} \mid z R_{i} x \text { for all } i \in N\right\}=\emptyset .
$$

If a profile of antisymmetric orderings $\left(R_{1}, \ldots, R_{n}\right)$ is such that $(1)$ and (2) are satisfied, we say that $\left(R_{1}, \ldots, R_{n}\right) \mathrm{END}_{n}$-rationalizes $C$.

The implications of requiring (1) alone are analyzed in Bossert and Sprumont (2001). Condition (2) by itself does not impose any restriction if $n \geq 2$ : for any selection function $C$, there exists a profile of antisymmetric orderings $\left(R_{1}, \ldots, R_{n}\right)$ such that $(2)$ is satisfiedjust let $R_{1}$ be an arbitrary antisymmetric ordering and let $R_{2}=\ldots=R_{n}$ be the inverse of $R_{1}$.

Combined with (1), (2) does impose additional restrictions. Consider the following example.

Example 1 Let $X=\{x, y, z\}, \mathcal{D}=\{(X, y),(X, z)\}, C(X, y)=\{x\}$ and $C(X, z)=\{y\}$. There exists a profile of antisymmetric orderings such that (1) is satisfied but any such profile must be such that $x R_{i} y$ for all $i \in N$; this is the case because $x$ is chosen when $y$ is the reference alternative and, consequently, non-deteriorating choice demands that everyone prefer $x$ to $y$. Because $x \in X$ and $C(X, z)=\{y\}$, this contradicts efficiency.

According to the traditional rational-choice model, an alternative $x$ is revealed preferred (in the weak sense; indifference is permitted) to an alternative $y$ if $x$ is chosen in a situation where $y$ is feasible. An analogous relation can be established in our framework. In contrast to the standard definition of the revealed-preference relation, a chosen alternative is not necessarily (weakly) preferred to all feasible alternatives but merely to the reference alternative. Thus, as in Bossert and Sprumont (2001), we can define a relation $R_{C}$ as follows: for all $x, y \in X$,

$$
x R_{C} y \Leftrightarrow \exists S \in \mathcal{P}(X) \text { such that }(S, y) \in \mathcal{D} \text { and } x \in C(S, y) \text {. }
$$

The following lemma is the counterpart of a result established by Samuelson $(1938,1948)$ in the standard revealed-preference framework; see also Richter (1971).

Lemma 1 Let $n \in I N$. If $\left(R_{1}, \ldots, R_{n}\right)$ END $D_{n}$-rationalizes $C$, then $\overline{R_{C}} \subseteq \bigcap_{i \in N} R_{i}$. 
Proof. Suppose $\left(R_{1}, \ldots, R_{n}\right) \mathrm{END}_{n}$-rationalizes $C$.

First, we show that $R_{C} \subseteq \bigcap_{i \in N} R_{i}$. Suppose $x R_{C} y$ for $x, y \in X$. Then there exists $S \in \mathcal{P}(X)$ such that $(S, y) \in \mathcal{D}$ and $x \in C(S, y)$. By part (a) of $\operatorname{END}_{n}$-rationalizability, this implies $x R_{i} y$ for all $i \in N$.

Now suppose $x \overline{R_{C}} y$ for $x, y \in X$. Then there exist $K \in \mathbb{N}$ and $x^{0}, \ldots, x^{K} \in X$ such that $x=x^{0}, x^{k-1} R_{C} x^{k}$ for all $k \in\{1, \ldots, K\}$ and $x^{K}=y$. Let $i \in N$. Because $R_{C} \subseteq R_{i}$, it follows that $x^{k-1} R_{i} x^{k}$ for all $k \in\{1, \ldots, K\}$. Because $R_{i}$ is transitive, this implies $x R_{i} y$. This is true for all $i \in N$, and the proof is complete.

Note that only (1) rather than the full requirement of $\mathrm{END}_{n}$-rationalizability is needed in the above result.

The following axiom is necessary for $\mathrm{END}_{n}$-rationalizability. It is also sufficient if, in a sense specified in the following theorem, $|X|$ is small relative to $n$. See Richter (1966) for a congruence axiom that is necessary and sufficient for rational choice in the traditional revealed-preference model.

END-Congruence: For all $x, y \in X$ and for all $(S, z) \in \mathcal{D}$, if $x \overline{R_{C}} y, x \neq y$ and $x \in S$, then $y \notin C(S, z)$.

We obtain

Theorem 1 Let $n \in \mathbb{N} \backslash\{1\}$.

(i) If $C$ is $E N D_{n}$-rationalizable, then $C$ satisfies END-congruence.

(ii) If $|X|<2(n+1)$ and $C$ satisfies END-congruence, then $C$ is $E N D_{n}$-rationalizable.

(iii) If $|X| \geq 2(n+1)$, then there exists a selection function $C$ satisfying ENDcongruence and violating $E N D_{n}$-rationalizability.

Proof. To prove part (i) of the theorem, suppose $C$ violates END-congruence. Then there exist $x, y \in X$ and $(S, z) \in \mathcal{D}$ such that $x \overline{R_{C}} y, x \neq y, x \in S$ and $y \in C(S, z)$. By Lemma $1, x R_{i} y$ for all $i \in N$ for any profile $\left(R_{1}, \ldots, R_{n}\right)$ that $\mathrm{END}_{n}$-rationalizes $C$, contradicting (2).

To prove part (ii), suppose $|X|<2(n+1)$ and $C$ satisfies END-congruence. Let $R^{0}=\overline{R_{C}} \cup R_{d}$. By definition, $R^{0}$ is a quasi-ordering.

Furthermore, $R^{0}$ is antisymmetric as a consequence of END-congruence. To prove this claim, suppose, by way of contradiction, that there exist two distinct alternatives $x, y \in X$ such that $x R^{0} y$ and $y R^{0} x$. By definition of $R^{0}$, this implies $x \overline{R_{C}} y$ and $y \overline{R_{C}} x$. Hence, there exist $K, L \in \mathbb{N}, x^{0}, \ldots, x^{K} \in X$ and $z^{0}, \ldots, z^{L} \in X$ such that $x=x^{0}, x^{k-1} R_{C} x^{k}$ for all 
$k \in\{1, \ldots, K\}, x^{K}=y, y=z^{0}, z^{\ell-1} R_{C} z^{\ell}$ for all $\ell \in\{1, \ldots, L\}$, and $z^{L}=x$. Without loss of generality, suppose $z^{L-1} \neq x$ (if not, replace $z^{L-1}$ with the highest-numbered $z^{\ell}$ that is different from $x$; this is always possible because $x \neq y$ ). By definition, we have $x \overline{R_{C}} z^{L-1}$. Because $z^{L-1} R_{C} z^{L}=x$, there exists $S \in \mathcal{P}(X)$ such that $(S, x) \in \mathcal{D}$ and $z^{L-1} \in C(S, x)$. But this contradicts END-congruence because $x \in S$.

Next, we prove that (1) and (2) hold if $R_{i}=R^{0}$ for all $i \in N$. (1) follows immediately by definition of $R_{C}$ and the fact that $R_{C} \subseteq R^{0}$. Now suppose, by way of contradiction, that (2) is not satisfied. Then there exist $(S, z) \in \mathcal{D}, x \in C(S, z)$ and $y \in S \backslash\{x\}$ such that $y R^{0} x$. But this contradicts END-congruence.

To continue the proof, the following definition is useful. The dimension of a quasiordering $R$ on $X$ is the smallest positive integer $r$ with the property that there exist $r$ orderings $R_{1}, \ldots, R_{r}$ whose intersection is $R$. For a real number $\alpha$, the largest integer less than or equal to $\alpha$ is denoted by $[\alpha]$.

We claim that the dimension of $R^{0}$ does not exceed $n$. If $|X| \leq 3$, the dimension of $R^{0}$ is less than or equal to two which, in turn, is less than or equal to $n$. If $|X| \geq 4$, Hiraguchi's inequality (see Hiraguchi, 1955) implies that the dimension of $R^{0}$ is less than or equal to $[|X| / 2]$, the largest integer less than or equal to $|X| / 2$. Because $|X|<2(n+1)$ implies $[|X| / 2] \leq n$, it follows again that the dimension of $R^{0}$ is less than or equal to $n$.

Thus, there exist antisymmetric orderings $R_{1}, \ldots, R_{n}$ (not necessarily distinct) on $X$ whose intersection is $R^{0}$. It is now straightforward to verify that the profile $\left(R_{1}, \ldots, R_{n}\right)$ $\mathrm{END}_{n}$-rationalizes $C$.

To prove part (iii) of the theorem, we adapt an example that was first developed in Sprumont (2001) in a different context.

Example 2 Suppose $|X| \geq 2(n+1)$. Let $\left\{a_{1}, \ldots, a_{n+1}, b_{1}, \ldots, b_{n+1}\right\} \subseteq X$. For convenience of notation, let $A=\left\{a_{1}, \ldots, a_{n+1}\right\}$ and $A_{-j}=A \backslash\left\{a_{j}\right\}$ for all $j \in\{1, \ldots, n+1\}$. Now define $C$ by letting

$$
\mathcal{D}=\cup_{j=1}^{n+1}\left\{\left(A \cup\left\{b_{j}\right\}, b_{j}\right),\left(\left\{a_{j}, b_{j}\right\}, b_{j}\right)\right\}
$$

and

$$
C\left(A \cup\left\{b_{j}\right\}, b_{j}\right)=A_{-j} \text { and } C\left(\left\{a_{j}, b_{j}\right\}, b_{j}\right)=\left\{b_{j}\right\} \text { for all } j \in\{1, \ldots, n+1\} \text {. }
$$

It is straightforward to verify that $C$ satisfies END-congruence. Next, by way of contradiction, suppose that $\left(R_{1}, \ldots, R_{n}\right)$ is a profile of antisymmetric orderings that $E N D_{n}$ rationalizes $C$. For all $i \in N$, let $x_{i}^{*}$ be the worst element in $\left\{a_{1}, \ldots, a_{n+1}\right\}$ according to $R_{i}$. 
Because the number of agents $n$ is less than $n+1$, there exists at least one $k \in\{1, \ldots, n+1\}$ such that $a_{k}$ is not a worst element for any $i \in N$. By definition,

$$
a_{k} R_{i} x_{i}^{*} \text { for all } i \in N \text {. }
$$

Note that $x_{i}^{*} \neq a_{k}$ for all $i \in N$. By (1), we have $y R_{i} b_{k}$ for all $y \in A_{-k}$ and for all $i \in N$. Therefore, in particular, $x_{i}^{*} R_{i} b_{k}$ for all $i \in N$. Together with (3), transitivity implies $a_{k} R_{i} b_{k}$ for all $i \in N$. Because $b_{k} \in C\left(\left\{a_{k}, b_{k}\right\}, b_{k}\right)$, this contradicts (2).

This completes the proof of the theorem.

An immediate corollary of part (ii) of the above theorem is that, if $C$ satisfies ENDcongruence and $X$ is finite, there must exist an $n \in \mathbb{N} \backslash\{1\}$ such that $C$ is $\operatorname{END}_{n^{-}}$ rationalizable. Furthermore, note that the assumption $n>1$ is required for part (ii) of the theorem only; clearly, parts (i) and (iii) are valid for $n=1$ as well. However, since this paper is concerned with multi-agent rationalizability, we do not state the obvious generalizations formally.

\section{The Universal Set Domain}

Consider the special case where, for every choice situation, the entire set $X$ is feasible. That is, for all $(S, y) \in \mathcal{D}, S=X$, and we can think of a selection function as a mapping that assigns a set of chosen alternatives to each reference alternative in its domain. Hence, to simplify notation, we now use a universal selection function, defined as a mapping $C_{X}: Y \rightarrow \mathcal{P}(X)$ where $Y \in \mathcal{P}(X)$. For every $y \in Y, C_{X}(y)$ is the set of selected alternatives in $X$ for the reference alternative $y$, that is, $C_{X}(y)=C(X, y)$ for all $y \in Y$. The definition of $\mathrm{END}_{n}$-rationalizability can be simplified as follows.

Universal $\mathbf{E N D}_{n}$-Rationalizability: There exists a profile $\left(R_{1}, \ldots, R_{n}\right)$ of antisymmetric orderings on $X$ such that

$$
x R_{i} y \text { for all } y \in Y \text {, for all } x \in C_{X}(y) \text { and for all } i \in N
$$

and

$$
\left\{y \in X \backslash\{x\} \mid y R_{i} x \text { for all } i \in N\right\}=\emptyset \text { for all } x \in C_{X}(Y) .
$$

If a profile of antisymmetric orderings $\left(R_{1}, \ldots, R_{n}\right)$ is such that (4) and (5) are satisfied for a universal selection function $C$, we say that $\left(R_{1}, \ldots, R_{n}\right)$ universally $\mathrm{END}_{n}$-rationalizes $C$. 
The counterpart of the relation $R_{C}$ defined in the previous section is denoted by $R_{C_{X}}$, that is,

$$
x R_{C_{X}} y \Leftrightarrow y \in Y \text { and } x \in C_{X}(y)
$$

for all $x, y \in X$. The following lemma is analogous to Lemma 1 .

Lemma 2 Let $n \in \mathbb{I N}$. If $\left(R_{1}, \ldots, R_{n}\right)$ universally END $D_{n}$-rationalizes $C_{X}$, then $R_{C_{X}} \subseteq$ $\bigcap_{i \in N} R_{i}$.

Proof. Suppose $x R_{C_{X}} y$. By definition, $y \in Y$ and $x \in C_{X}(y)$, and part (a) of universal $\mathrm{END}_{n}$-rationalizability implies $x R_{i} y$ for all $i \in N$.

Again, the full force of the universal rationalizability condition is not needed - all that is required is (4). Because we restrict attention to problems where the feasible set is always the entire universal set $X$, the relation $R_{C_{X}}$ turns out to be transitive as a consequence of a suitable formulation of the END-congruence axiom for $C_{X}$. We may therefore simplify this axiom as follows.

Universal END-Congruence: For all $x, y \in X$, if $x R_{C_{X}} y$ and $x \neq y$, then $y \notin C_{X}(Y)$.

With the domain assumption considered in this section, it turns out that this axiom is necessary and sufficient for $\mathrm{END}_{n}$-rationalizability for any value of $n \in \mathbb{N} \backslash\{1\}$ and for any cardinality of $X$. We obtain

Theorem 2 Let $n \in \mathbb{I} \backslash\{1\} . C_{X}$ is universally $E N D_{n}$-rationalizable if and only if $C_{X}$ satisfies universal END-congruence.

Proof. "Only if." Suppose $C_{X}$ violates universal END-congruence. Then there exist $x, y \in X$ such that $x R_{C_{X}} y, x \neq y$ and $y \in C_{X}(Y)$. By Lemma 2, $x R_{i} y$ for all $i \in N$, contradicting (5).

"If." Suppose $C_{X}$ satisfies universal END-congruence. Define the relation $Q$ on $X$ by letting, for all $x, y \in X$,

$$
x Q y \Leftrightarrow x=y \text { or }\left[x \in C_{X}(Y) \text { and } y \in X \backslash C_{X}(Y)\right] .
$$

That $Q$ is reflexive is immediate. Note that, for all $x, y \in X$ such that $x Q y$ and $x \neq y$, we have $x \in C_{X}(Y)$ and $y \in X \backslash C_{X}(Y)$. This observation immediately implies that $Q$ is transitive and antisymmetric. Furthermore, we have $R_{C_{X}} \subseteq Q$ by universal ENDcongruence. 
Let $R$ be an arbitrary antisymmetric ordering on $X \backslash C_{X}(Y)$, and define $R^{0}=Q \cup R$. Again, $R^{0}$ is a reflexive, transitive and antisymmetric relation such that $R_{C_{X}} \subseteq Q \subseteq R^{0}$. According to the relation $R^{0}$, the noncomparable pairs $(x, y)$ are all such that both $x$ and $y$ are in $C_{X}(Y)$.

Let $R_{1}^{0}$ be an arbitrary antisymmetric ordering on $C_{X}(Y)$, and let $R_{2}^{0}=\ldots=R_{n}^{0}$ be the inverse of $R_{1}^{0}$. Define

$$
R_{i}=R^{0} \cup R_{i}^{0}
$$

for all $i \in N$. It is straightforward to verify that the $R_{i}$ are antisymmetric orderings. It remains to be shown that the profile $\left(R_{1}, \ldots, R_{n}\right)$ universally $\operatorname{END}_{n}$-rationalizes $C_{X}$. That (4) is satisfied follows immediately from the observation that $R_{C_{X}} \subseteq R_{i}$ for all $i \in N$. To prove (5), suppose $x \in C_{X}(Y)$. This implies $x Q y$ and thus $x R_{i} y$ for all $y \in X \backslash C_{X}(Y)$ and for all $i \in N$ and, thus, no $y \in X \backslash C_{X}(Y)$ can Pareto dominate $x$. Furthermore, we have

$$
x R_{1} y \text { or } x R_{2} y
$$

for all $y \in C_{X}(Y) \backslash\{x\}$ by definition and, thus, $x$ cannot be Pareto dominated by another alternative in $C_{X}(Y)$ either.

\section{Concluding Remarks}

We conclude with a few remarks on some questions related to those discussed in this paper. First, an obvious open problem is to find necessary and sufficient conditions for $\mathrm{END}_{n}$-rationalizability on arbitrary domains. In general, the existence of a profile that $\mathrm{END}_{n}$-rationalizes a selection function depends on the value of $n$, and the problem of identifying necessary and sufficient conditions leads to a mathematical exercise that is closely related to the problem of determining the dimension of an ordering; see, for example, Dushnik and Miller (1941). Because this is an area that is still quite unsettled, it is not too surprising that tight results are hard to come by in our framework as well. One reason why we are able to identify necessary and sufficient conditions for universal set domains is that, due to the assumption that the entire universal set $X$ is feasible for all selection problems, all multi-agent notions of $\mathrm{END}_{n}$-rationalizability collapse into a single requirement: $\mathrm{END}_{n}$-rationalizability is equivalent to $\mathrm{END}_{m}$-rationalizability for all $n, m \in \mathbb{N} \backslash\{1\}$.

Another related issue concerns the application of the notion of collective rationality introduced here to more structured environments similar to those considered by Chiappori 
(1988) involving, for instance, private or public goods. While necessary and sufficient conditions for non-deteriorating choice and for efficient choice when treated separately are known in the case where $X$ contains allocations of consumption bundles, the combination of the two requirements is substantially more complex. Even for the counterpart of the universal set domain in an exchange economy, characterization results are difficult to obtain. One problem that arises is that, unlike in the abstract case where preferences are essentially unrestricted, the usual economic assumptions on individual preferences such as strict monotonicity and strict convexity prevent us from employing the inverse of an arbitrary individual preference relation. Thus, the construction of rationalizing profiles employed here does not translate into that environment.

\section{References}

ARrow, K.J. (1959), "Rational choice functions and orderings," Economica 26, 121-127.

Banerjee, A. and Pattanaik, P.K. (1996), "A note on a property of maximal sets and choice in the absence of universal comparability," Economics Letters 51, 191-195.

Bossert, W. and Sprumont, Y. (2001), "Non-deteriorating choice," Discussion Paper 01-2001, C.R.D.E., Université de Montréal.

Bossert, W. And Sprumont, Y. (2002), "Core rationalizability in two-agent exchange economies," Economic Theory 20, 777-791.

Brown, D. And Matzkin, R. (1996), "Testable restrictions on the equilibrium manifold," Econometrica 64, 1249-1262.

Chiappori, P.-A. (1988), "Rational household labor supply," Econometrica 56, 63-89.

Dushnik, B. AND Miller, E.W. (1941), "Partially ordered sets," American Journal of Mathematics 63, 600-610.

Hiraguchi, T. (1955), "On the dimension of orders," Scientific Report Kanazawa University 4, 1-20.

Hansson, B. (1968), "Choice structures and preference relations," Synthese 18, 443-458.

Masatli, Y. AND OK, E.A. (2002), "Rational choice with a status quo bias," mimeo, Department of Economics, New York University. 
RAY, I. ANd Zhou, L. (2001), "Game theory via revealed preferences," Games and Economic Behavior 37, 415-424.

RIChteR, M.K. (1966), "Revealed preference theory," Econometrica 34, 635-645.

Richter, M.K. (1971), "Rational choice," in J. Chipman, L. Hurwicz, M. Richter, And H. Sonnenschein (eds.), Preferences, Utility, and Demand, Harcourt Brace Jovanovich, New York, pp. 29-58.

SAmuelson, P.A. (1938), "A note on the pure theory of consumer's behaviour," Economica $5,61-71$.

SAmuelson, P.A. (1948), "Consumption theory in terms of revealed preference," Economica 15, 243-253.

SEn, A.K. (1971), "Choice functions and revealed preference," Review of Economic Studies 38, 307-317.

Sprumont, Y. (2000), "On the testable implications of collective choice theories," Journal of Economic Theory 93, 205-232.

Sprumont, Y. (2001), "Paretian quasi-orders: the regular two-agent case," Journal of Economic Theory 101, 437-456.

Zhou, L. (1997), "Revealed preferences: the role of the status quo," mimeo, Department of Economics, Duke University. 